\title{
Acceleration of the Halpern algorithm to search for a fixed point of a nonexpansive mapping
}

\author{
Kaito Sakurai* and Hideaki liduka
}

"Correspondence:

k_sakurai@cs.meiji.ac.jp

Department of Computer Science,

Meiji University, 1-1-1 Higashimita,

Tama-ku, Kawasaki-shi, Kanagawa

214-8571, Japan

\begin{abstract}
This paper presents an algorithm to accelerate the Halpern fixed point algorithm in a real Hilbert space. To this goal, we first apply the Halpern algorithm to the smooth convex minimization problem, which is an example of a fixed point problem for a nonexpansive mapping, and indicate that the Halpern algorithm is based on the steepest descent method for solving the minimization problem. Next, we formulate a novel fixed point algorithm using the ideas of conjugate gradient methods that can accelerate the steepest descent method. We show that, under certain assumptions, our algorithm strongly converges to a fixed point of a nonexpansive mapping. We numerically compare our algorithm with the Halpern algorithm and show that it dramatically reduces the running time and iterations needed to find a fixed point compared with that algorithm.
\end{abstract}

MSC: $47 \mathrm{H} 10 ; 65 \mathrm{~K} 05 ; 90 \mathrm{C} 25$

Keywords: conjugate gradient method; fixed point; Halpern algorithm; nonexpansive mapping; smooth convex optimization; steepest descent method

\section{Introduction}

Fixed point problems for nonexpansive mappings [1, Chapter 4], [2, Chapter 3], [3, Chapter 1], [4, Chapter 3] have been investigated in many practical applications, and they include convex feasibility problems [5], [1, Example 5.21], convex optimization problems [1, Corollary 17.5], problems of finding the zeros of monotone operators [1, Proposition 23.38], and monotone variational inequalities [1, Subchapter 25.5].

Fixed point problems can be solved by using useful fixed point algorithms, such as the Krasnosel'skiu-Mann algorithm [1, Subchapter 5.2], [6, Subchapter 1.2], [7, 8], the Halpern algorithm [6, Subchapter 1.2], [9, 10], and the hybrid method [11]. Meanwhile, to make practical systems and networks (see, e.g., [12-15] and references therein) stable and reliable, the fixed point has to be found at a faster pace. That is, we need a new algorithm that approximates the fixed point faster than the conventional ones. In this paper, we focus on the Halpern algorithm and present an algorithm to accelerate the search for a fixed point of a nonexpansive mapping.

To achieve the main objective of this paper, we first apply the Halpern algorithm to the smooth convex minimization problem, which is an example of a fixed point problem for

\section{空 Springer}

@2014 Sakurai and liduka; licensee Springer. This is an Open Access article distributed under the terms of the Creative Commons Attribution License (http://creativecommons.org/licenses/by/2.0), which permits unrestricted use, distribution, and reproduction in any medium, provided the original work is properly cited. 
a nonexpansive mapping, and indicate that the Halpern algorithm is based on the steepest descent method [16, Subchapter 3.3] for solving the minimization problem.

A number of iterative methods [16, Chapters 5-19] have been proposed to accelerate the steepest descent method. In particular, conjugate gradient methods [16, Chapter 5] have been widely used as an efficient accelerated version of most gradient methods. Here, we focus on the conjugate gradient methods and devise an algorithm blending the conjugate gradient methods with the Halpern algorithm.

Our main contribution is to propose a novel algorithm for finding a fixed point of a nonexpansive mapping, for which we use the ideas of accelerated conjugate gradient methods for optimization over the fixed point set [17, 18], and prove that the algorithm converges to some fixed point in the sense of the strong topology of a real Hilbert space. To demonstrate the effectiveness and fast convergence of our algorithm, we numerically compare our algorithm with the Halpern algorithm. Numerical results show that it dramatically reduces the running time and iterations needed to find a fixed point compared with that algorithm.

This paper is organized as follows. Section 2 gives the mathematical preliminaries. Section 3 devises the acceleration algorithm for solving fixed point problems and presents its convergence analysis. Section 4 applies the proposed and conventional algorithms to a concrete fixed point problem and provides numerical examples comparing them.

\section{Mathematical preliminaries}

Let $H$ be a real Hilbert space with the inner product $\langle\cdot, \cdot\rangle$ and its induced norm $\|\cdot\|$, and let $\mathbb{N}$ be the set of all positive integers including zero.

\subsection{Fixed point problem}

Suppose that $C \subset H$ is nonempty, closed, and convex. A mapping $T: C \rightarrow C$ is said to be nonexpansive [1, Definition 4.1(ii)], [2, (3.2)], [3, Subchapter 1.1], [4, Subchapter 3.1] if

$$
\|T(x)-T(y)\| \leq\|x-y\| \quad(x, y \in C) .
$$

The fixed point set of $T: C \rightarrow C$ is denoted by

$$
\operatorname{Fix}(T):=\{x \in C: T(x)=x\} .
$$

The metric projection onto $C$ [1, Subchapter 4.2, Chapter 28$]$ is denoted by $P_{C}$. It is defined by $P_{C}(x) \in C$ and $\left\|x-P_{C}(x)\right\|=\inf _{y \in C}\|x-y\|(x \in H)$. $P_{C}$ is nonexpansive with Fix $\left(P_{C}\right)=C$ [1, Proposition 4.8, (4.8)].

Proposition 2.1 Suppose that $C \subset H$ is nonempty, closed, and convex, $T: C \rightarrow C$ is nonexpansive, and $x \in H$. Then

(i) [1, Corollary 4.15], [2, Lemma 3.4], [3, Proposition 5.3], [4, Theorem 3.1.6] Fix $(T)$ is closed and convex.

(ii) [1, Theorem 3.14] $\hat{x}=P_{C}(x)$ if and only if $\langle x-\hat{x}, y-\hat{x}\rangle \leq 0(y \in C)$.

Proposition 2.1(i) guarantees that if $\operatorname{Fix}(T) \neq \emptyset, P_{\operatorname{Fix}(T)}(x)$ exists for all $x \in H$.

This paper discusses the following fixed point problem. 
Problem 2.1 Suppose that $T: H \rightarrow H$ is nonexpansive with $\operatorname{Fix}(T) \neq \emptyset$. Then

find $x^{\star} \in H$ such that $T\left(x^{\star}\right)=x^{\star}$.

\subsection{The Halpern algorithm and our algorithm}

The Halpern algorithm generates the sequence $\left(x_{n}\right)_{n \in \mathbb{N}}[6$, Subchapter 1.2] $[9,10]$ as follows: given $x_{0} \in H$ and $\left(\alpha_{n}\right)_{n \in \mathbb{N}}$ satisfying $\lim _{n \rightarrow \infty} \alpha_{n}=0, \sum_{n=0}^{\infty} \alpha_{n}=\infty$, and $\sum_{n=0}^{\infty} \mid \alpha_{n+1}-$ $\alpha_{n} \mid<\infty$

$$
x_{n+1}:=\alpha_{n} x_{0}+\left(1-\alpha_{n}\right) T\left(x_{n}\right) \quad(n \in \mathbb{N}) .
$$

Algorithm (1) strongly converges to $P_{\mathrm{Fix}(T)}\left(x_{0}\right)(\in \operatorname{Fix}(T))$ [6, Theorem 6.17], [9, 10].

Here, we shall discuss Problem 2.1 when Fix $(T)$ is the set of all minimizers of a convex, continuously Fréchet differentiable functional $f$ over $H$ and see that algorithm (1) is based on the steepest descent method [16, Subchapter 3.3] to minimize $f$ over $H$. Suppose that the gradient of $f$, denoted by $\nabla f$, is Lipschitz continuous with a constant $L>0$ and define $T^{f}: H \rightarrow H$ by

$$
T^{f}:=I-\alpha \nabla f
$$

where $\alpha \in(0,2 / L]$ and $I: H \rightarrow H$ stands for the identity mapping. Accordingly, $T^{f}$ satisfies the nonexpansivity condition (see, e.g., [12, Proposition 2.3]) and

$$
\operatorname{Fix}\left(T^{f}\right)=\underset{x \in H}{\operatorname{argmin}} f(x):=\left\{x^{\star} \in H: f\left(x^{\star}\right)=\min _{x \in H} f(x)\right\} .
$$

Therefore, algorithm (1) with $T:=T^{f}$ can be expressed as follows.

$$
\left\{\begin{array}{l}
d_{n+1}^{f}:=-\nabla f\left(x_{n}\right), \\
y_{n}:=T^{f}\left(x_{n}\right)=x_{n}-\alpha \nabla f\left(x_{n}\right)=x_{n}+\alpha d_{n+1}^{f}, \\
x_{n+1}:=\alpha_{n} x_{0}+\left(1-\alpha_{n}\right) y_{n} \quad(n \in \mathbb{N}) .
\end{array}\right.
$$

This implies that algorithm (3) uses the steepest descent direction [16, Subchapter 3.3] $d_{n+1}^{f, S D D}:=-\nabla f\left(x_{n}\right)$ of $f$ at $x_{n}$, and hence algorithm (3) is based on the steepest descent method.

Meanwhile, conjugate gradient methods [16, Chapter 5] are popular acceleration methods of the steepest descent method. The conjugate gradient direction of $f$ at $x_{n}(n \in \mathbb{N})$ is $d_{n+1}^{f, \mathrm{CGD}}:=-\nabla f\left(x_{n}\right)+\beta_{n} d_{n}^{f, \mathrm{CGD}}$, where $d_{0}^{f, \mathrm{CGD}}:=-\nabla f\left(x_{0}\right)$ and $\left(\beta_{n}\right)_{n \in \mathbb{N}} \subset(0, \infty)$, which, together with (2), implies that

$$
d_{n+1}^{f, \mathrm{CGD}}=\frac{1}{\alpha}\left(T^{f}\left(x_{n}\right)-x_{n}\right)+\beta_{n} d_{n}^{f, \mathrm{CGD}} .
$$

Therefore, by replacing $d_{n+1}^{f}:=-\nabla f\left(x_{n}\right)$ in algorithm (3) with $d_{n+1}^{f, \text { CGD }}$ defined by (4), we can formulate a novel algorithm for solving Problem 2.1.

Before presenting the algorithm, we provide the following lemmas which are used to prove the main theorem. 
Proposition 2.2 [6, Lemmas 1.2 and 1.3] Let $\left(a_{n}\right)_{n \in \mathbb{N}},\left(b_{n}\right)_{n \in \mathbb{N}},\left(c_{n}\right)_{n \in \mathbb{N}},\left(\alpha_{n}\right)_{n \in \mathbb{N}} \subset(0, \infty)$ be sequences with $a_{n+1} \leq\left(1-\alpha_{n}\right) a_{n}+\alpha_{n} b_{n}+c_{n}(n \in \mathbb{N})$. Suppose that $\sum_{n=0}^{\infty} \alpha_{n}=\infty$, $\lim \sup _{n \rightarrow \infty} b_{n} \leq 0$, and $\sum_{n=0}^{\infty} c_{n}<\infty$. Then $\lim _{n \rightarrow \infty} a_{n}=0$.

Proposition 2.3 [19, Lemma 1] Suppose that $\left(x_{n}\right)_{n \in \mathbb{N}} \subset H$ weakly converges to $x \in H$ and $y \neq x$. Then $\liminf _{n \rightarrow \infty}\left\|x_{n}-x\right\|<\liminf _{n \rightarrow \infty}\left\|x_{n}-y\right\|$.

\section{Acceleration of the Halpern algorithm}

We present the following algorithm.

\section{Algorithm 3.1}

Step 0. Choose $\mu \in(0,1], \alpha>0$, and $x_{0} \in H$ arbitrarily, and set $\left(\alpha_{n}\right)_{n \in \mathbb{N}} \subset(0,1),\left(\beta_{n}\right)_{n \in \mathbb{N}} \subset$ $[0, \infty)$. Compute $d_{0}:=\left(T\left(x_{0}\right)-x_{0}\right) / \alpha$.

Step 1. Given $x_{n}, d_{n} \in H$, compute $d_{n+1} \in H$ by

$$
d_{n+1}:=\frac{1}{\alpha}\left(T\left(x_{n}\right)-x_{n}\right)+\beta_{n} d_{n} .
$$

Compute $x_{n+1} \in H$ as follows.

$$
\left\{\begin{array}{l}
y_{n}:=x_{n}+\alpha d_{n+1}, \\
x_{n+1}:=\mu \alpha_{n} x_{0}+\left(1-\mu \alpha_{n}\right) y_{n} .
\end{array}\right.
$$

Put $n:=n+1$, and go to Step 1 .

We can check that Algorithm 3.1 coincides with the Halpern algorithm (1) when $\beta_{n}:=0$ $(n \in \mathbb{N})$ and $\mu:=1$.

This section makes the following assumptions.

Assumption 3.1 The sequences $\left(\alpha_{n}\right)_{n \in \mathbb{N}}$ and $\left(\beta_{n}\right)_{n \in \mathbb{N}}$ satisfy $^{\mathrm{a}}$
(C1) $\lim _{n \rightarrow \infty} \alpha_{n}=0$
(C2) $\sum_{n=0}^{\infty} \alpha_{n}=\infty$
(C3) $\sum_{n=0}^{\infty}\left|\alpha_{n+1}-\alpha_{n}\right|<\infty$,

(C4) $\beta_{n} \leq \alpha_{n}^{2} \quad(n \in \mathbb{N})$.

Moreover, $\left(x_{n}\right)_{n \in \mathbb{N}}$ in Algorithm 3.1 satisfies

(C5) $\left(T\left(x_{n}\right)-x_{n}\right)_{n \in \mathbb{N}}$ is bounded.

Let us do a convergence analysis of Algorithm 3.1.

Theorem 3.1 Under Assumption 3.1, the sequence $\left(x_{n}\right)_{n \in \mathbb{N}}$ generated by Algorithm 3.1 strongly converges to $P_{\mathrm{Fix}(T)}\left(x_{0}\right)$.

Algorithm 3.1 when $\beta_{n}:=0(n \in \mathbb{N})$ is the Halpern algorithm defined by

$$
x_{n+1}:=\mu \alpha_{n} x_{0}+\left(1-\mu \alpha_{n}\right) T\left(x_{n}\right) \quad(n \in \mathbb{N}) .
$$


Hence, the nonexpansivity of $T$ ensures that, for all $x \in \operatorname{Fix}(T)$ and for all $n \in \mathbb{N}$,

$$
\begin{aligned}
\left\|x_{n+1}-x\right\| & =\left\|\mu \alpha_{n}\left(x_{0}-x\right)+\left(1-\mu \alpha_{n}\right)\left(T\left(x_{n}\right)-x\right)\right\| \\
& \leq \mu \alpha_{n}\left\|x_{0}-x\right\|+\left(1-\mu \alpha_{n}\right)\left\|T\left(x_{n}\right)-x\right\| \\
& \leq \mu \alpha_{n}\left\|x_{0}-x\right\|+\left(1-\mu \alpha_{n}\right)\left\|x_{n}-x\right\| .
\end{aligned}
$$

Suppose that $n:=0$. From (5), we have $\left\|x_{1}-x\right\| \leq \mu \alpha_{0}\left\|x_{0}-x\right\|+\left(1-\mu \alpha_{0}\right)\left\|x_{0}-x\right\|=\left\|x_{0}-x\right\|$. Assume that $\left\|x_{m}-x\right\| \leq\left\|x_{0}-x\right\|$ for some $m \in \mathbb{N}$. Then (5) implies that $\left\|x_{m+1}-x\right\| \leq$ $\mu \alpha_{m}\left\|x_{0}-x\right\|+\left(1-\mu \alpha_{m}\right)\left\|x_{m}-x\right\| \leq \mu \alpha_{m}\left\|x_{0}-x\right\|+\left(1-\mu \alpha_{m}\right)\left\|x_{0}-x\right\|=\left\|x_{0}-x\right\|$. Hence, induction guarantees that

$$
\left\|x_{n}-x\right\| \leq\left\|x_{0}-x\right\| \quad(n \in \mathbb{N})
$$

Therefore, we find that $\left(x_{n}\right)_{n \in \mathbb{N}}$ is bounded. Moreover, since the nonexpansivity of $T$ ensures that $\left(T\left(x_{n}\right)\right)_{n \in \mathbb{N}}$ is also bounded, (C5) holds. Accordingly, Theorem 3.1 says that if $\left(\alpha_{n}\right)_{n \in \mathbb{N}}$ satisfies $(\mathrm{C} 1)-(\mathrm{C} 3)$, Algorithm 3.1 when $\beta_{n}:=0(n \in \mathbb{N})$ (i.e., the Halpern algorithm) strongly converges to $P_{\mathrm{Fix}(T)}\left(x_{0}\right)$. This means that Theorem 3.1 is a generalization of the convergence analysis of the Halpern algorithm.

\subsection{Proof of Theorem 3.1}

We first show the following lemma.

Lemma 3.1 Suppose that Assumption 3.1 holds. Then $\left(d_{n}\right)_{n \in \mathbb{N}},\left(x_{n}\right)_{n \in \mathbb{N}}$, and $\left(y_{n}\right)_{n \in \mathbb{N}}$ are bounded.

Proof We have from (C1) and (C4) that $\lim _{n \rightarrow \infty} \beta_{n}=0$. Accordingly, there exists $n_{0} \in \mathbb{N}$ such that $\beta_{n} \leq 1 / 2$ for all $n \geq n_{0}$. Define $M_{1}:=\max \left\{\left\|d_{n_{0}}\right\|,(2 / \alpha) \sup _{n \in \mathbb{N}}\left\|T\left(x_{n}\right)-x_{n}\right\|\right\}$. Then (C5) implies that $M_{1}<\infty$. Assume that $\left\|d_{n}\right\| \leq M_{1}$ for some $n \geq n_{0}$. The triangle inequality ensures that

$$
\left\|d_{n+1}\right\|=\left\|\frac{1}{\alpha}\left(T\left(x_{n}\right)-x_{n}\right)+\beta_{n} d_{n}\right\| \leq \frac{1}{\alpha}\left\|T\left(x_{n}\right)-x_{n}\right\|+\beta_{n}\left\|d_{n}\right\| \leq M_{1},
$$

which means that $\left\|d_{n}\right\| \leq M_{1}$ for all $n \geq n_{0}$, i.e., $\left(d_{n}\right)_{n \in \mathbb{N}}$ is bounded.

The definition of $y_{n}(n \in \mathbb{N})$ implies that

$$
\begin{aligned}
y_{n} & =x_{n}+\alpha\left(\frac{1}{\alpha}\left(T\left(x_{n}\right)-x_{n}\right)+\beta_{n} d_{n}\right) \\
& =T\left(x_{n}\right)+\alpha \beta_{n} d_{n} .
\end{aligned}
$$

The nonexpansivity of $T$ and (6) imply that, for all $x \in \operatorname{Fix}(T)$ and for all $n \geq n_{0}$,

$$
\begin{aligned}
\left\|y_{n}-x\right\| & =\left\|T\left(x_{n}\right)+\alpha \beta_{n} d_{n}-x\right\| \\
& \leq\left\|T\left(x_{n}\right)-T(x)\right\|+\alpha \beta_{n}\left\|d_{n}\right\| \\
& \leq\left\|x_{n}-x\right\|+\alpha M_{1} \beta_{n} .
\end{aligned}
$$


Therefore, we find that, for all $x \in \operatorname{Fix}(T)$ and for all $n \geq n_{0}$,

$$
\begin{aligned}
\left\|x_{n+1}-x\right\| & =\left\|\mu \alpha_{n}\left(x_{0}-x\right)+\left(1-\mu \alpha_{n}\right)\left(y_{n}-x\right)\right\| \\
& \leq \mu \alpha_{n}\left\|x_{0}-x\right\|+\left(1-\mu \alpha_{n}\right)\left\|y_{n}-x\right\| \\
& \leq \mu \alpha_{n}\left\|x_{0}-x\right\|+\left(1-\mu \alpha_{n}\right)\left\{\left\|x_{n}-x\right\|+\alpha M_{1} \beta_{n}\right\} \\
& \leq\left(1-\mu \alpha_{n}\right)\left\|x_{n}-x\right\|+\mu \alpha_{n}\left\|x_{0}-x\right\|+\alpha M_{1} \beta_{n}
\end{aligned}
$$

which, together with (C4) and $\alpha_{n}<1(n \in \mathbb{N})$, means that, for all $x \in \operatorname{Fix}(T)$ and for all $n \geq n_{0}$,

$$
\left\|x_{n+1}-x\right\| \leq\left(1-\mu \alpha_{n}\right)\left\|x_{n}-x\right\|+\mu \alpha_{n}\left(\left\|x_{0}-x\right\|+\frac{\alpha M_{1}}{\mu}\right)
$$

Induction guarantees that, for all $x \in \operatorname{Fix}(T)$ and for all $n \geq n_{0}$,

$$
\left\|x_{n}-x\right\| \leq\left\|x_{0}-x\right\|+\frac{\alpha M_{1}}{\mu}
$$

Therefore, $\left(x_{n}\right)_{n \in \mathbb{N}}$ is bounded.

The definition of $y_{n}(n \in \mathbb{N})$ and the boundedness of $\left(x_{n}\right)_{n \in \mathbb{N}}$ and $\left(d_{n}\right)_{n \in \mathbb{N}}$ imply that $\left(y_{n}\right)_{n \in \mathbb{N}}$ is also bounded. This completes the proof.

Lemma 3.2 Suppose that Assumption 3.1 holds. Then

(i) $\lim _{n \rightarrow \infty}\left\|x_{n+1}-x_{n}\right\|=0$.

(ii) $\lim _{n \rightarrow \infty}\left\|x_{n}-T\left(x_{n}\right)\right\|=0$.

(iii) $\limsup _{n \rightarrow \infty}\left\langle x_{0}-x^{\star}, y_{n}-x^{\star}\right\rangle \leq 0$, where $x^{\star}:=P_{\operatorname{Fix}(T)}\left(x_{0}\right)$.

Proof (i) Equation (6), the triangle inequality, and the nonexpansivity of $T$ imply that, for all $n \in \mathbb{N}$,

$$
\begin{aligned}
\left\|y_{n+1}-y_{n}\right\| & =\left\|T\left(x_{n+1}\right)-T\left(x_{n}\right)+\alpha\left(\beta_{n+1} d_{n+1}-\beta_{n} d_{n}\right)\right\| \\
& \leq\left\|T\left(x_{n+1}\right)-T\left(x_{n}\right)\right\|+\alpha\left\|\beta_{n+1} d_{n+1}-\beta_{n} d_{n}\right\| \\
& \leq\left\|x_{n+1}-x_{n}\right\|+\alpha\left(\beta_{n+1}\left\|d_{n+1}\right\|+\beta_{n}\left\|d_{n}\right\|\right),
\end{aligned}
$$

which, together with $\left\|d_{n}\right\| \leq M_{1}\left(n \geq n_{0}\right)$ and (C4), implies that, for all $n \geq n_{0}$,

$$
\left\|y_{n+1}-y_{n}\right\| \leq\left\|x_{n+1}-x_{n}\right\|+\alpha M_{1}\left(\alpha_{n+1}^{2}+\alpha_{n}^{2}\right) \text {. }
$$

On the other hand, from $\alpha_{n} \leq\left|\alpha_{n+1}-\alpha_{n}\right|+\alpha_{n+1}$ and $\alpha_{n}<1(n \in \mathbb{N})$, we have that, for all $n \in \mathbb{N}$,

$$
\begin{aligned}
\alpha_{n+1}^{2}+\alpha_{n}^{2} & \leq \alpha_{n+1}^{2}+\alpha_{n}\left(\left|\alpha_{n+1}-\alpha_{n}\right|+\alpha_{n+1}\right) \\
& \leq\left(\alpha_{n+1}+\alpha_{n}\right) \alpha_{n+1}+\left|\alpha_{n+1}-\alpha_{n}\right|
\end{aligned}
$$


We also find that, for all $n \in \mathbb{N} \backslash\{0\}$,

$$
\begin{aligned}
\left\|x_{n+1}-x_{n}\right\|= & \left\|\mu \alpha_{n} x_{0}+\left(1-\mu \alpha_{n}\right) y_{n}-\left(\mu \alpha_{n-1} x_{0}+\left(1-\mu \alpha_{n-1}\right) y_{n-1}\right)\right\| \\
= & \| \mu\left(\alpha_{n}-\alpha_{n-1}\right) x_{0}+\left(1-\mu \alpha_{n}\right)\left(y_{n}-y_{n-1}\right) \\
& +\mu\left(\alpha_{n-1}-\alpha_{n}\right) y_{n-1} \| \\
\leq & \mu\left|\alpha_{n}-\alpha_{n-1}\right|\left(\left\|x_{0}\right\|+\left\|y_{n-1}\right\|\right)+\left(1-\mu \alpha_{n}\right)\left\|y_{n}-y_{n-1}\right\| \\
\leq & \left(1-\mu \alpha_{n}\right)\left\|y_{n}-y_{n-1}\right\|+M_{2}\left|\alpha_{n}-\alpha_{n-1}\right|,
\end{aligned}
$$

where $M_{2}:=\sup _{n \in \mathbb{N}} \mu\left(\left\|x_{0}\right\|+\left\|y_{n}\right\|\right)<\infty$. Hence, (7) and (8) ensure that, for all $n \geq n_{0}$,

$$
\begin{aligned}
\left\|x_{n+1}-x_{n}\right\| \leq & \left(1-\mu \alpha_{n}\right)\left\|x_{n}-x_{n-1}\right\|+\alpha M_{1}\left(\left(\alpha_{n}+\alpha_{n-1}\right) \alpha_{n}+\left|\alpha_{n}-\alpha_{n-1}\right|\right) \\
& +M_{2}\left|\alpha_{n}-\alpha_{n-1}\right| \\
= & \left(1-\mu \alpha_{n}\right)\left\|x_{n}-x_{n-1}\right\|+\left(\alpha M_{1}+M_{2}\right)\left|\alpha_{n}-\alpha_{n-1}\right| \\
& +\frac{\alpha M_{1}}{\mu}\left(\alpha_{n}+\alpha_{n-1}\right) \mu \alpha_{n} .
\end{aligned}
$$

Proposition 2.2, (C1), (C2), and (C3) lead us to

$$
\lim _{n \rightarrow \infty}\left\|x_{n+1}-x_{n}\right\|=0 .
$$

(ii) From $\left\|x_{n+1}-y_{n}\right\|=\mu \alpha_{n}\left\|x_{0}-y_{n}\right\| \leq M_{2} \alpha_{n}(n \in \mathbb{N})$, (C1) means that $\lim _{n \rightarrow \infty} \| x_{n+1}-$ $y_{n} \|=0$. Since the triangle inequality ensures that $\left\|y_{n}-x_{n}\right\| \leq\left\|y_{n}-x_{n+1}\right\|+\left\|x_{n+1}-x_{n}\right\|$ $(n \in \mathbb{N})$, we find from (9) that

$$
\lim _{n \rightarrow \infty}\left\|d_{n+1}\right\|=\frac{1}{\alpha} \lim _{n \rightarrow \infty}\left\|y_{n}-x_{n}\right\|=0
$$

From the definition of $d_{n+1}(n \in \mathbb{N})$, we have, for all $n \geq n_{0}$,

$$
0 \leq \frac{1}{\alpha}\left\|T\left(x_{n}\right)-x_{n}\right\| \leq\left\|d_{n+1}\right\|+\beta_{n}\left\|d_{n}\right\| \leq\left\|d_{n+1}\right\|+M_{1} \beta_{n} .
$$

Since Equation (10) and $\lim _{n \rightarrow \infty} \beta_{n}=0$ guarantee that the right-hand side of the above inequality converges to 0 , we find that

$$
\lim _{n \rightarrow \infty}\left\|T\left(x_{n}\right)-x_{n}\right\|=0
$$

(iii) From the limit superior of $\left(\left\langle x_{0}-x^{\star}, y_{n}-x^{\star}\right\rangle\right)_{n \in \mathbb{N}}$, there exists $\left(y_{n_{k}}\right)_{k \in \mathbb{N}}\left(\subset\left(y_{n}\right)_{n \in \mathbb{N}}\right)$ such that

$$
\limsup _{n \rightarrow \infty}\left\langle x_{0}-x^{\star}, y_{n}-x^{\star}\right\rangle=\lim _{k \rightarrow \infty}\left\langle x_{0}-x^{\star}, y_{n_{k}}-x^{\star}\right\rangle .
$$

Moreover, since $\left(y_{n_{k}}\right)_{k \in \mathbb{N}}$ is bounded, there exists $\left(y_{n_{k_{k}}}\right)_{i \in \mathbb{N}}\left(\subset\left(y_{n_{k}}\right)_{k \in \mathbb{N}}\right)$ which weakly converges to some point $\hat{y}(\in H)$. Equation (10) guarantees that $\left(x_{n_{k_{i}}}\right)_{i \in \mathbb{N}}$ weakly converges to $\hat{y}$. 
We shall show that $\hat{y} \in \operatorname{Fix}(T)$. Assume that $\hat{y} \notin \operatorname{Fix}(T)$, i.e., $\hat{y} \neq T(\hat{y})$. Proposition 2.3, (11), and the nonexpansivity of $T$ ensure that

$$
\begin{aligned}
\liminf _{i \rightarrow \infty}\left\|x_{n_{k_{i}}}-\hat{y}\right\| & <\liminf _{i \rightarrow \infty}\left\|x_{n_{k_{i}}}-T(\hat{y})\right\| \\
& =\liminf _{i \rightarrow \infty}\left\|x_{n_{k_{i}}}-T\left(x_{n_{k_{i}}}\right)+T\left(x_{n_{k_{i}}}\right)-T(\hat{y})\right\| \\
& =\liminf _{i \rightarrow \infty}\left\|T\left(x_{n_{k_{i}}}\right)-T(\hat{y})\right\| \\
& \leq \liminf _{i \rightarrow \infty}\left\|x_{n_{k_{i}}}-\hat{y}\right\| .
\end{aligned}
$$

This is a contradiction. Hence, $\hat{y} \in \operatorname{Fix}(T)$. Hence, (12) and Proposition 2.1(ii) guarantee that

$$
\limsup _{n \rightarrow \infty}\left\langle x_{0}-x^{\star}, y_{n}-x^{\star}\right\rangle=\lim _{i \rightarrow \infty}\left\langle x_{0}-x^{\star}, y_{n_{k_{i}}}-x^{\star}\right\rangle=\left\langle x_{0}-x^{\star}, \hat{y}-x^{\star}\right\rangle \leq 0 .
$$

This completes the proof.

Now, we are in a position to prove Theorem 3.1.

Proof of Theorem 3.1 The inequality $\|x+y\|^{2} \leq\|x\|^{2}+2\langle y, x+y\rangle(x, y \in H)$, (6), and the nonexpansivity of $T$ imply that, for all $n \in \mathbb{N}$,

$$
\begin{aligned}
\left\|y_{n}-x^{\star}\right\|^{2} & =\left\|T\left(x_{n}\right)-x^{\star}+\alpha \beta_{n} d_{n}\right\|^{2} \\
& \leq\left\|T\left(x_{n}\right)-T\left(x^{\star}\right)\right\|^{2}+2 \alpha \beta_{n}\left\langle y_{n}-x^{\star}, d_{n}\right\rangle \\
& \leq\left\|x_{n}-x^{\star}\right\|^{2}+M_{3} \alpha_{n}^{2},
\end{aligned}
$$

where $\beta_{n} \leq \alpha_{n}^{2}(n \in \mathbb{N})$ and $M_{3}:=\sup _{n \in \mathbb{N}} 2 \alpha\left|\left\langle y_{n}-x^{\star}, d_{n}\right\rangle\right|<\infty$. We thus have that, for all $n \in \mathbb{N}$,

$$
\begin{aligned}
\left\|x_{n+1}-x^{\star}\right\|^{2}= & \left\|\mu \alpha_{n}\left(x_{0}-x^{\star}\right)+\left(1-\mu \alpha_{n}\right)\left(y_{n}-x^{\star}\right)\right\|^{2} \\
= & \mu^{2} \alpha_{n}^{2}\left\|x_{0}-x^{\star}\right\|^{2}+\left(1-\mu \alpha_{n}\right)^{2}\left\|y_{n}-x^{\star}\right\|^{2} \\
& +2 \mu \alpha_{n}\left(1-\mu \alpha_{n}\right)\left(x_{0}-x^{\star}, y_{n}-x^{\star}\right\rangle \\
\leq & \mu^{2} \alpha_{n}^{2}\left\|x_{0}-x^{\star}\right\|^{2}+\left(1-\mu \alpha_{n}\right)^{2}\left\{\left\|x_{n}-x^{\star}\right\|^{2}+M_{3} \alpha_{n}^{2}\right\} \\
& +2 \mu \alpha_{n}\left(1-\mu \alpha_{n}\right)\left(x_{0}-x^{\star}, y_{n}-x^{\star}\right\rangle \\
\leq & \left(1-\mu \alpha_{n}\right)\left\|x_{n}-x^{\star}\right\|^{2}+\left\{\mu \alpha_{n}\left\|x_{0}-x^{\star}\right\|^{2}+\frac{M_{3} \alpha_{n}}{\mu}\right\} \mu \alpha_{n} \\
& +\left\{2\left(1-\mu \alpha_{n}\right)\left(x_{0}-x^{\star}, y_{n}-x^{\star}\right)\right\} \mu \alpha_{n} .
\end{aligned}
$$

Proposition 2.2, (C1), (C2), and Lemma 3.2(iii) lead one to deduce that

$$
\lim _{n \rightarrow \infty}\left\|x_{n+1}-x^{\star}\right\|^{2}=0
$$


This guarantees that $\left(x_{n}\right)_{n \in \mathbb{N}}$ generated by Algorithm 3.1 strongly converges to $x^{\star}:=$ $P_{\mathrm{Fix}(T)}\left(x_{0}\right)$.

Suppose that $\operatorname{Fix}(T)$ is bounded. Then we can set a bounded, closed convex set $C$ ( $\supset$ Fix $(T)$ ) such that $P_{C}$ can be computed within a finite number of arithmetic operations (e.g., $C$ is a closed ball with a large enough radius). Hence, we can compute

$$
x_{n+1}:=P_{C}\left(\mu \alpha_{n} x_{0}+\left(1-\mu \alpha_{n}\right) y_{n}\right)
$$

instead of $x_{n+1}$ in Algorithm 3.1. From $\left(x_{n}\right)_{n \in \mathbb{N}} \subset C$, the boundedness of $C$ means that $\left(x_{n}\right)_{n \in \mathbb{N}}$ is bounded. The nonexpansivity of $T$ guarantees that $\left\|T\left(x_{n}\right)-T(x)\right\| \leq\left\|x_{n}-x\right\|$ $(x \in \operatorname{Fix}(T))$, which means that $\left(T\left(x_{n}\right)\right)_{n \in \mathbb{N}}$ is bounded. Therefore, $(C 5)$ holds. We can prove that Algorithm 3.1 with (13) strongly converges to a point in $\operatorname{Fix}(T)$ by referring to the proof of Theorem 3.1.

Let us consider the case where $\operatorname{Fix}(T)$ is unbounded. In this case, we cannot choose a bounded $C$ satisfying $\operatorname{Fix}(T) \subset C$. Although we can execute Algorithm 3.1, we need to verify the boundedness of $\left(T\left(x_{n}\right)-x_{n}\right)_{n \in \mathbb{N}}$. Instead, we can apply the Halpern algorithm (1) to this case without any problem. However, the Halpern algorithm would converge slowly because it is based on the steepest descent method (see Section 1). Hence, in this case, it would be desirable to execute not only the Halpern algorithm but also Algorithm 3.1.

\section{Numerical examples and conclusion}

Let us apply the Halpern algorithm (1) and Algorithm 3.1 to the following convex feasibility problem [5], [1, Example 5.21].

Problem 4.1 Given a nonempty, closed convex set $C_{i} \subset \mathbb{R}^{N}(i=0,1, \ldots, m)$,

$$
\text { find } x^{\star} \in C:=\bigcap_{i=0}^{m} C_{i} \text {, }
$$

where one assumes that $C \neq \varnothing$.

Define a mapping $T: \mathbb{R}^{N} \rightarrow \mathbb{R}^{N}$ by

$$
T:=P_{0}\left(\frac{1}{m} \sum_{i=1}^{m} P_{i}\right) \text {, }
$$

where $P_{i}:=P_{C_{i}}(i=0,1, \ldots, m)$ stands for the metric projection onto $C_{i}$. Since $P_{i}(i=$ $0,1, \ldots, m)$ is nonexpansive, $T$ defined by (14) is also nonexpansive. Moreover, we find that

$$
\operatorname{Fix}(T)=\operatorname{Fix}\left(P_{0}\right) \cap \bigcap_{i=1}^{m} \operatorname{Fix}\left(P_{i}\right)=C_{0} \cap \bigcap_{i=1}^{m} C_{i}=C .
$$

Therefore, Problem 4.1 coincides with Problem 2.1 with $T$ defined by (14).

The experiment used an Apple Macbook Air with a $1.30 \mathrm{GHz}$ Intel(R) Core(TM) i5-4250U CPU and 4GB DDR3 memory. The Halpern algorithm (1) and Algorithm 3.1 were written in Java. The operating system of the computer was Mac OSX version 10.8.5. 
Figure 1 Behavior of $\left\|T\left(x_{n}\right)-x_{n}\right\|$ for the Halpern algorithm and Algorithm 3.1 (proposed). (The Halpern algorithm took 850 iterations to satisfy $\left\|T\left(x_{n}\right)-x_{n}\right\|<10^{-6}$, whereas Algorithm 3.1 took only six.)

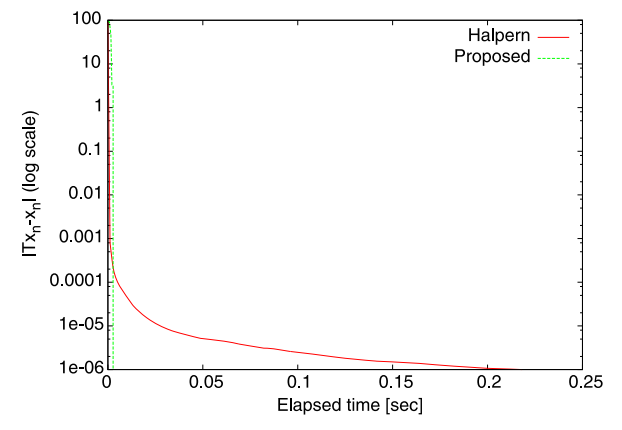

We set $\alpha:=1, \mu:=1 / 10^{5}, \alpha_{n}:=1 /(n+1)(n \in \mathbb{N})$, and $\beta_{n}:=1 /(n+1)^{2}(n \in \mathbb{N})$ in Algorithm 3.1 and compared Algorithm 3.1 with the Halpern algorithm (1) with $\alpha_{n}:=\mu /(n+1)$ $(n \in \mathbb{N})$. In the experiment, we set $C_{i}(i=0,1, \ldots, m)$ as a closed ball with center $c_{i} \in \mathbb{R}^{N}$ and radius $r_{i}>0$. Thus, $P_{i}(i=0,1, \ldots, m)$ can be computed with

$$
P_{i}(x):=c_{i}+\frac{r_{i}}{\left\|c_{i}-x\right\|}\left(x-c_{i}\right) \quad \text { if }\left\|c_{i}-x\right\|>r_{i}
$$

or $P_{i}(x):=x$ if $\left\|c_{i}-x\right\| \leq r_{i}$.

We set $N:=100, m:=3, r_{i}:=1(i=0,1,2,3)$, and $c_{0}:=0$. The experiment used random vectors $c_{i} \in(-1 / \sqrt{N}, 1 / \sqrt{N})^{N}(i=1,2,3)$ generated by the java.util.Random class so as to satisfy $C \neq \emptyset$. We also used the java.util.Random class to set a random initial point in $(-16,16)^{N}$.

Figure 1 describes the behaviors of $\left\|T\left(x_{n}\right)-x_{n}\right\|$ for the Halpern algorithm (1) and Algorithm 3.1 (proposed). The $x$-axis and $y$-axis represent the elapsed time and value of $\left\|T\left(x_{n}\right)-x_{n}\right\|$. The results show that compared with the Halpern algorithm, Algorithm 3.1 dramatically reduces the time required to satisfy $\left\|T\left(x_{n}\right)-x_{n}\right\|<10^{-6}$. We found that the Halpern algorithm took 850 iterations to satisfy $\left\|T\left(x_{n}\right)-x_{n}\right\|<10^{-6}$, whereas Algorithm 3.1 took only six.

This paper presented an algorithm to accelerate the Halpern algorithm for finding a fixed point of a nonexpansive mapping on a real Hilbert space and its convergence analysis. The convergence analysis guarantees that the proposed algorithm strongly converges to a fixed point of a nonexpansive mapping under certain assumptions. We numerically compared the abilities of the proposed and Halpern algorithms in solving a concrete fixed point problem. The results showed that the proposed algorithm performs better than the Halpern algorithm.

Competing interests

The authors declare that they have no competing interests.

Authors' contributions

All authors contributed equally to the writing of this paper. All authors read and approved the final manuscript.

\section{Acknowledgements}

We are sincerely grateful to the associate editor Lai-Jiu Lin and the two anonymous reviewers for helping us improve the original manuscript.

Endnote

${ }^{a}$ Examples of $\left(\alpha_{n}\right)_{n \in \mathbb{N}}$ and $\left(\beta_{n}\right)_{n \in \mathbb{N}}$ satisfying (C1)-(C4) are $\alpha_{n}:=1 /(n+1)^{a}$ and $\beta_{n}:=1 /(n+1)^{2 a}(n \in \mathbb{N})$, where $a \in(0,1]$. 


\section{References}

1. Bauschke, HH, Combettes, PL: Convex Analysis and Monotone Operator Theory in Hilbert Spaces. Springer, Berlin (2011)

2. Goebel, K, Kirk, WA: Topics in Metric Fixed Point Theory. Cambridge Studies in Advanced Mathematics. Cambridge University Press, Cambridge (1990)

3. Goebel, K, Reich, S: Uniform Convexity, Hyperbolic Geometry, and Nonexpansive Mappings. Dekker, New York (1984)

4. Takahashi, W: Nonlinear Functional Analysis. Yokohama Publishers, Yokohama (2000)

5. Bauschke, HH, Borwein, JM: On projection algorithms for solving convex feasibility problems. SIAM Rev. 38(3), 367-426 (1996)

6. Berinde, V: Iterative Approximation of Fixed Points. Springer, Berlin (2007)

7. Krasnosel'skiĬ, MA: Two remarks on the method of successive approximations. Usp. Mat. Nauk 10, 123-127 (1955)

8. Mann, WR: Mean value methods in iteration. Proc. Am. Math. Soc. 4, 506-510 (1953)

9. Halpern, B: Fixed points of nonexpansive maps. Bull. Am. Math. Soc. 73, 957-961 (1967)

10. Wittmann, R: Approximation of fixed points of nonexpansive mappings. Arch. Math. 58(5), 486-491 (1992)

11. Nakajo, K, Takahashi, W: Strong convergence theorems for nonexpansive mappings and nonexpansive semigroups. J. Math. Anal. Appl. 279, 372-379 (2003)

12. liduka, $\mathrm{H}$ : Iterative algorithm for solving triple-hierarchical constrained optimization problem. J. Optim. Theory Appl. 148, 580-592 (2011)

13. liduka, H: Fixed point optimization algorithm and its application to power control in CDMA data networks. Math. Program. 133, 227-242 (2012)

14. liduka, $\mathrm{H}$ : Iterative algorithm for triple-hierarchical constrained nonconvex optimization problem and its application to network bandwidth allocation. SIAM J. Optim. 22(3), 862-878 (2012)

15. liduka, H: Fixed point optimization algorithms for distributed optimization in networked systems. SIAM J. Optim. 23 $1-26(2013)$

16. Nocedal, J, Wright, SJ: Numerical Optimization, 2nd edn. Springer Series in Operations Research and Financial Engineering. Springer, Berlin (2006)

17. liduka, $\mathrm{H}$ : Acceleration method for convex optimization over the fixed point set of a nonexpansive mapping. Math. Program. (2014). doi:10.1007/s10107-013-0741-1

18. liduka, H, Yamada, I: A use of conjugate gradient direction for the convex optimization problem over the fixed point set of a nonexpansive mapping. SIAM J. Optim. 19(4), 1881-1893 (2009)

19. Opial, Z: Weak convergence of the sequence of successive approximation for nonexpansive mappings. Bull. Am. Math. Soc. 73, 591-597 (1967)

10.1186/1687-1812-2014-202

Cite this article as: Sakurai and liduka: Acceleration of the Halpern algorithm to search for a fixed point of a nonexpansive mapping. Fixed Point Theory and Applications 2014, 2014:202

\section{Submit your manuscript to a SpringerOpen ${ }^{\circ}$ journal and benefit from:}

- Convenient online submission

- Rigorous peer review

- Immediate publication on acceptance

- Open access: articles freely available online

- High visibility within the field

- Retaining the copyright to your article 